\title{
A parallel domain decomposition-based implicit method for the Cahn-Hilliard-Cook phase-field equation in 3D
}

\author{
Xiang Zheng ${ }^{\mathrm{a}}$, Chao Yang ${ }^{\mathrm{b}, \mathrm{e}}$, Xiao-Chuan Cai ${ }^{\mathrm{c}, *}$, David Keyes ${ }^{\mathrm{a}, \mathrm{d}}$ \\ ${ }^{a}$ Department of Applied Physics and Applied Mathematics, Columbia University, New York, NY \\ 10027, USA \\ ${ }^{b}$ Institute of Software, Chinese Academy of Sciences, Beijing 100190, China \\ ${ }^{c}$ Department of Computer Science, University of Colorado Boulder, Boulder, CO 80309, USA \\ ${ }^{d}$ Computer, Electrical and Mathematical Sciences and Engineering, King Abdullah University of \\ Science and Technology, Jeddah 21534, Saudi Arabia \\ ${ }^{e}$ State Key Laboratory of Computer Science, Chinese Academy of Sciences, Beijing 100190,
}

China

\begin{abstract}
We present a numerical algorithm for simulating the spinodal decomposition described by the three dimensional Cahn-Hilliard-Cook (CHC) equation, which is a fourth-order stochastic partial differential equation with a noise term. The equation is discretized in space and time based on a fully implicit, cell-centered finite difference scheme, with an adaptive time-stepping strategy designed to accelerate the progress to equilibrium. At each time step, a parallel Newton-Krylov-Schwarz algorithm is used to solve the nonlinear system. We discuss various numerical and computational challenges associated with the method. The numerical scheme is validated by a comparison with an explicit scheme of high accuracy (and unreasonably high cost). We present steady state solutions of the CHC equation in two and three dimensions. The effect of the thermal fluctuation on the spinodal decomposition process is studied. We show that the existence of the thermal fluctuation accelerates the spinodal decomposition process and that the final steady morphology is sensitive to the stochastic noise. We also show the evolution of the energies and statistical moments. In terms of the parallel performance, it is found that the implicit domain decomposition approach scales well on supercomputers with a large number of processors.
\end{abstract}

\footnotetext{
${ }^{*}$ Corresponding author

Email address: cai@cs.colorado.edu (Xiao-Chuan Cai)
} 
Keywords: Cahn-Hilliard-Cook, thermal fluctuation, implicit method, Newton-Krylov-Schwarz, parallel scalability, steady state solutions.

\section{Introduction}

Proposed in 1958, the Cahn-Hilliard $(\mathrm{CH})$ equation was initially used to model phase transition in a binary alloy $[8,9]$. The $\mathrm{CH}$ equation has subsequently found many applications from nanoscale [43] to planetary-scale [36], such as polymers [33], image processing [17], electromagnetic driven void migration [41], and planetary dynamics [36]. Since the pioneering work of Langer et al. [30], numerical methods for the $\mathrm{CH}$ equation have been extensively studied, including finite difference [14, 34], finite element [21, 22, 42], finite volume [16, 28], and Monte-Carlo lattice methods [31].

Recently progress has been made to simulate the 3D morphological evolution of the $\mathrm{CH}$ equation. With a NURBS-based finite element scheme and implicit adaptive time stepping, Gomez et al. performed an isogeometric analysis of the $\mathrm{CH}$ equation and obtained from early random initial conditions several steady morphologies in both 2D and 3D [24]. By using a domain decomposition method for parallel computing, Wodo et al. performed further analysis on various implicit schemes and identified all five hypothesized periodic solutions of the $\mathrm{CH}$ equation in 3D [37]. Yang et al. conducted similar studies using thousands of processor cores, and discovered that lower-order boundary conditions in the additive Schwarz preconditioner lead to better convergence of the implicit solver $[39,40]$.

In 1970, Cook found that thermal fluctuations play an important role in the process of spinodal decomposition [15] and the $\mathrm{CH}$ equation may be unrealistic for describing the entire process, especially for the early state [4]. Cook proposed to revise the $\mathrm{CH}$ equation by including the influence of the thermal fluctuation, which is also known as the Cahn-Hilliard-Cook (CHC) equation [15]. Since then numerous papers in the literature have addressed the modeling and effects of the thermal fluctuation $[4,5,19,20]$. Due to the existence of the thermal fluctuation in spinodal decomposition experiments, only a stochastic process can correctly describe the whole physical process [3]. Recently Galenko et al. suggested that the diffusion field (the deterministic contribution) and the noise (the stochastic contribution) both have an essential influence on the rate of spinodal decomposition [23].

There are a number of published numerical results on the CHC equation. Hawick et al. discussed various discretization schemes for integrating the $\mathrm{CHC}$ system, 
and the visualization of the morphology evolution [25]. Based on the CHC equation, Rogers et al. studied numerically the effect of the thermal fluctuation in the early stage, and the domain growth for a critical quench in the late stage in two dimensions $[19,20]$. Using a finite difference method, Cardon-Weber developed an implicit scheme for the $\mathrm{CHC}$ equation, which converges in the sense of probability [13]. Kovács et al. introduced a finite element method for the CHC equation, and showed its optimal order of convergence [29]. The high spatial order and the noise in the $\mathrm{CHC}$ equation make the numerical study very expensive, especially in 3D. Moreover, the energy of the solution, a principal quantity of interest, changes by leaps between near plateaus, which makes numerical integration challenging.

In this work we propose an efficient parallel algorithm for computing the equilibrium solutions of the $\mathrm{CHC}$ equation. The equation is discretized by a fully implicit, cell-centered finite difference scheme; the time step size is decided by an adaptive time stepping strategy; the conserved noise term is approximated by a random variable with a Gaussian distribution. A Newton-Krylov-Schwarz algorithm is used to solve the nonlinear problem at each time step $[10,11,26]$. Compared with explicit methods, the fully implicit scheme allows much larger time steps. We are able to compute the entire evolution of the morphologies, energies, and statistical moments in both $2 \mathrm{D}$ and $3 \mathrm{D}$. It is found that the thermal fluctuation accelerates the spinodal decomposition process. We compare the final steady morphology obtained with the $\mathrm{CHC}$ and the $\mathrm{CH}$ equations, and find that the stochastic noise changes the final steady morphology in some situations.

The remainder of the paper is organized as follows. The next section offers some physical background about the $\mathrm{CHC}$ equation. The numerical methodology and solvers are presented in Section 3, and numerical results with some analysis are given in Section 4. Finally, Section 5 provides concluding remarks and possible future research directions.

\section{Physical background}

In this section we briefly describe the physical phenomena and a mathematical derivation of the CHC equation. Spinodal decomposition, as well as other phase separation process, starts from an initially homogeneous mixture, evolves through spontaneous growth of fluctuations of concentration, and results in the separations with distinctly different chemical compositions [39]. For a binary mixture system, we define the local concentration of the two species as $c_{1}$ and $c_{2}$, with $c_{1}+c_{2}=1$ as a result of mass conservation. In this paper we use the concentration difference $u=c_{1}-c_{2} \in[-1,1]$ as the dependent variable in the phase field model. Here 
$u$ is a function of $x \in \Omega$ and time $t \geq 0$. In particular, $u= \pm 1$ represents pure fluids at equilibrium of the spinodal decomposition. A unit cubic domain $\Omega$ and periodic boundary conditions are considered in our study.

Based on the Ginzburg-Landau theory, the energy function for a binary mixture system typically consists of two parts, i.e., $E(u)=E_{1}(u)+E_{2}(u)$. Here $E_{1}(u)=\int_{\Omega} f(u) d \Omega$ is the bulk energy and $E_{2}(u)=\int_{\Omega} \frac{\epsilon^{2}}{2}|\nabla u|^{2} d \Omega$ is the interfacial energy ( $\epsilon>0$ is called the interfacial parameter). The bulk energy is dependent only on the local concentration difference. And the interfacial energy, which is scaled by $\epsilon$, depends on the concentration gradient.

The potential term $f(u)$ in the bulk energy has a non-convex and double-well form, and is often approximated by the polynomial function $f(u)=1 / 4\left(1-u^{2}\right)^{2}$. However, the above quartic form is inaccurate when the phase quench is deep, i.e., the ratio between the critical temperature and the absolute temperature is large [2]. Using a more realistic Flory-Huggins model [37], the potential term takes the logarithmic form:

$$
f(u)=\frac{1}{2}\left((1+u) \ln (1+u)+(1-u) \ln (1-u)-\theta u^{2}\right),
$$

where $\theta$ is the ratio of the critical temperature $T_{c}$ and the temperature $T$. For $\theta>1$, the non-convex chemical free energy has a double-well form and drives the phase separation into two binodal points, while for $\theta \leq 1$, it has only one well and generates a single phase. Following [24], we take the quench ratio $\theta=3 / 2$, corresponding to a physically relevant case.

The dissipation of the Cahn-Hilliard energy is:

$$
J(u)=\frac{\delta E(u)}{\delta u} .
$$

The system evolves to the equilibrium by minimizing the free energy. Taking into account of the thermal fluctuation, the mathematical form of the spinodal decomposition process is

$$
\frac{\partial u}{\partial t}=-\nabla \cdot M(u) \nabla J(u)+\zeta(u)
$$

where $\zeta(u)$ is the thermal contribution to the total flux [7], and $M(u)$ is the mobility. In this paper we employ a variable mobility $M=1 / 4\left(1-u^{2}\right)$ for the thermodynamic consistency. This dependence between the mobility and the concentration difference produces important changes during the coarsening process $[24,27]$. 
Combining (1) - (2) into (3), the CHC equation becomes:

$$
\frac{\partial u}{\partial t}+\left(\epsilon^{2} \nabla \cdot M \nabla(\Delta u)+\theta \nabla \cdot M \nabla u-\frac{1}{4} \Delta u\right)+\zeta=0
$$

We model the thermal fluctuation using the Langevin approach [19, 35, 38], which assumes that $\zeta$ is a Gaussian space-time white noise, with the following properties:

$$
\left\{\begin{array}{l}
\langle\zeta(t, x)\rangle=0, \\
\left\langle\zeta\left(t_{1}, x_{1}\right) \zeta\left(t_{2}, x_{2}\right)\right\rangle=-\sigma M \delta\left(t_{1}-t_{2}\right) \Delta \delta\left(x_{1}-x_{2}\right),
\end{array}\right.
$$

where $\sqrt{\sigma}$ is the intensity of the thermal fluctuation, $\delta()$ is the Dirac Delta function, and \langle\rangle is the expectation operator defined as $\langle\eta\rangle=\int_{-\infty}^{+\infty} \eta p(\eta) d \eta$, where $\eta$ is a random variable and $p$ is the probability density function.

We remark that the statistical conditions of the thermal fluctuation imply three things [25]. First, there is no overall drift force. Second, the random noise is uncorrelated in time, but partially correlated in space, which escapes long wave length components in the noise spectrum. Finally, as a result of the Laplacian operator, a conservation law is satisfied by the random force. A random force component which piles up matter at one cell center must be exactly balanced by force contributions at neighboring cells that deplete the matter. The second condition of (5) ensures this property.

\section{Numerical methodology and solvers}

In this section, we illustrate our numerical methodology in $3 \mathrm{D}$, while numerical results of both $2 \mathrm{D}$ and $3 \mathrm{D}$ are shown in the next section. The CHC equation (4) is a high-order parabolic equation, thus explicit schemes are limited in terms of time step size due to stability restrictions. In order to use large time steps and maintain good accuracy, we discretize (4) with a fully implicit, cell-centered finite difference scheme. A nonlinear system is solved by using the Newton-KrylovSchwarz method at each time step.

\subsection{Discretization}

Without loss of generality, we discretize the CHC equation on a unit cube, i.e., $\Omega=[0,1]^{3}$. We use a uniform mesh with $N=1 / h$ mesh cells in each direction and formally denote $x_{i}=(i-0.5) h, y_{j}=(j-0.5) h, z_{k}=(k-0.5) h$ as the center of the cell. In finite difference scheme, the solution $u$ is approximated as $u_{i j k} \approx u\left(x_{i}, y_{j}, z_{k}\right)$, for all $1 \leq i, j, k \leq N$. The discretized solution on the time 
level $t=t^{n}$ is denoted as $u_{i j k}^{n}$, and $t^{0}=0$ is the initial time level. We use the notation

$$
\nabla_{h}=\left(D_{x}, D_{y}, D_{z}\right)
$$

to represent the discrete gradient operator, where

$$
D_{x} u_{i j k}=\frac{u_{i+\frac{1}{2} j k}-u_{i-\frac{1}{2} j k}}{h},
$$

and $D_{y}$, and $D_{z}$ are defined similarly, with a natural generalization to nonuniform Cartesian meshes in which $n$ is defined locally for each coordinate. Then the Laplacian operator $\Delta$ is discretized by

$$
\Delta_{h}=\nabla_{h} \cdot \nabla_{h}=D_{x}^{2}+D_{y}^{2}+D_{z}^{2},
$$

and $\nabla \cdot M \nabla$ is discretized by

$$
(\nabla \cdot M \nabla)_{h}=\nabla_{h} \cdot M \nabla_{h}=D_{x} M D_{x}+D_{y} M D_{y}+D_{z} M D_{z},
$$

where the value of $M$ on a cell face is approximated by the averaged value of $M$ on the two adjacent cell centers. With the above discretization, the stencil for cell $(i, j, k)$ depends on the values from all mesh cells $\left(i^{\prime}, j^{\prime}, k^{\prime}\right)$ satisfying $\left|i^{\prime}-i\right|+\left|j^{\prime}-j\right|+\left|k^{\prime}-k\right| \leq 2$. Thus the overall scheme is a 25-point stencil. In this paper, we use the periodic boundary condition.

The condition in (5) has the discrete form $[19,35]$

$$
\left\{\begin{array}{l}
\left\langle\zeta_{i j k}^{n}\right\rangle=0, \\
\left\langle\zeta_{i j k}^{n} \zeta_{i^{\prime} j^{\prime} k^{\prime}}^{n^{\prime}}\right\rangle=-\sigma M\left(u_{i j k}^{n}\right) \frac{\delta_{n n^{\prime}}}{\left|t^{n}-t^{n^{\prime}}\right|} \Delta_{h} \frac{\delta_{(i j k)\left(i^{\prime} j^{\prime} k^{\prime}\right)}}{h^{3}},
\end{array}\right.
$$

where $\zeta_{i j k}^{n}$ is the discretized thermal fluctuation at $(i, j, k)$ of the $n^{t h}$ time level $t^{n}$, $\delta_{i j}$ is the Kronecker delta, with single in time or multi-index in space. If $i$ and $j$ are component-wise equal, then $\delta_{i j}=1$, otherwise $\delta_{i j}=0$.

In our numerical examples, we replace the discretized thermal fluctuation $\zeta_{i j k}^{n}$ by a series of computer-generated pseudo independent random numbers $\left\{\xi_{:} \xi_{i}, i=\right.$ $\left.1,2, \cdots, 3 N^{3}\right\}$ obeying an uniform distribution in $[0,1]$. We pair $\xi$ into $3 N^{3} / 2$ subgroups. For each subgroup, there are two independent random numbers $\xi_{i}$ and $\xi_{i+1}$. The Box-Mueller algorithm transforms them into two independent random numbers $\rho_{i}$ and $\rho_{i+1}$ satisfying the standard Gaussian distribution [6]:

$$
\left\{\begin{array}{c}
\rho_{i}=\sqrt{-2 \ln \xi_{i}} \cos \left(2 \pi \xi_{i+1}\right) \\
\rho_{i+1}=\sqrt{-2 \ln \xi_{i}} \sin \left(2 \pi \xi_{i+1}\right) .
\end{array}\right.
$$


Therefore, using $\xi$ we obtain a series of independent random numbers $\{\rho$ : $\left.\rho_{i}, i=1,2, \cdots, 3 N^{3}\right\}$ that satisfy $\left\langle\rho_{i}\right\rangle=0$ and $\left\langle\rho_{i} \rho_{i^{\prime}}\right\rangle=\delta_{i i^{\prime}}$. For each $(i, j, k)$, we need a vector including three independent random numbers: $\boldsymbol{\rho}_{i j k}^{n}=\left(\rho_{i j k x}, \rho_{i j k y}, \rho_{i j k z}\right)$, where $(i j k x, i j k y, i j k z)=\left(3(i-1) N^{2}+3(j-1) N+3(k-1)\right) \times(1,1,1)+(1,2,3)$ is the mapping from the one dimensional series $\xi$ to the three dimensional vectors $\boldsymbol{\rho}_{i j k}^{n}$.

We express $\zeta_{i j k}^{n}$ by $\boldsymbol{\rho}_{i j k}^{n}$ and $\nabla_{h}[35]$

$$
\zeta_{i j k}^{n}=\sqrt{\frac{\sigma M\left(u_{i j k}^{n}\right)}{h^{3} \Delta t^{n}}} \nabla_{h} \cdot \boldsymbol{\rho}_{i j k}^{n},
$$

where $\Delta t^{n}=t^{n}-t^{n-1}$ is the time step size at the $n^{\text {th }}$ time level. We should note that, by making the vectors $\boldsymbol{\rho}_{i j k}^{n}$ at each cell of each time step independent, independent $\xi$ are generated at each $t^{n}$.

We employ a backward Euler scheme for the time integration, i.e., the spatial derivative term and the thermal fluctuation term are both evaluated at the $n^{\text {th }}$ time level, and the transient term is approximated as

$$
\left(\frac{\partial u_{i j k}^{n}}{\partial t}\right)_{h}=\frac{u_{i j k}^{n}-u_{i j k}^{n-1}}{\Delta t^{n}},
$$

with first-order accuracy.

With the fully implicit discretization scheme, we need to solve a system of nonlinear algebraic equations

$$
\mathbf{F}\left(\mathbf{u}^{n}\right):=\frac{\mathbf{u}^{n}-\mathbf{u}^{n-1}}{\Delta t^{n}}+G\left(\mathbf{u}^{n}\right)+\boldsymbol{\zeta}_{h}^{n}=0
$$

where $\mathbf{F}=\left(F_{000}^{n}, \ldots, F_{i j k}^{n}, \ldots\right)^{\mathrm{T}}$ and $\mathbf{u}^{n}=\left(u_{000}^{n}, \ldots, u_{i j k}^{n}, \ldots\right)^{\mathrm{T}}$. In (10), $G$ represents the spatial discretization, and $\zeta_{h}^{n}$ is the discretized thermal fluctuation.

The evolution of a phase-field problem admits various time scales. For a typical spinodal decomposition, the minimization of the chemical energy results in very fast development in the early stage, and later in the coarsening process, the dissipation of the interfacial energy is orders of magnitude slower. An adaptive time-step control is necessary in the numerical simulation. The idea of the adaptive time-stepping is from $[24,37]$, which works well for the Cahn-Hilliard equation. We extend the idea by using the variation of the solution $u$ of two consecutive time steps as the monitoring criteria. In our case the time step size is computed by

$$
\Delta t^{n}=\min \left\{\alpha \Delta t^{n-1}, \Delta t_{\max }\right\},
$$


when $n>1$ and where

$$
\alpha=\min \left\{\alpha_{\max }, \max \left\{\alpha_{\min },\left(1+\gamma \cdot\left(\rho_{0} \frac{\left\|\mathbf{u}^{n-1}\right\|+\beta}{\left\|\mathbf{u}^{n-1}-\mathbf{u}^{n-2}\right\|}-1\right)\right)\right\}\right\} .
$$

Here $\gamma, \beta$ and $\rho_{0}$ are used to control the degree of adaptivity as in [24, 37]. Some safeguards, such as $\alpha_{\max }, \alpha_{\min }$, and $\Delta t_{\max }$ are included to avoid excessive increase or decrease of the time step size. The values of the above parameters are selected experimentally. In this paper, we set $\Delta t_{\max }=0.1, \alpha_{\max }=1.5, \alpha_{\min }=0.6$, $\gamma=0.5, \beta=3.0, \rho_{0}=0.015$, and an initial time step size $\Delta t_{0}=1.5 \times 10^{-4}$.

\subsection{Newton-Krylov-Schwarz solvers}

For solving (10), a Newton-Krylov-Schwarz (NKS) method is applied. The NKS method has three main components: (1) an inexact Newton method for solving the nonlinear system, with the solution of the previous time step as the initial guess; (2) a Krylov subspace method for solving the linear Jacobian system at each Newton iteration; and (3) a Schwarz preconditioner for the Jacobian matrix.

The inexact Newton method solves (10) iteratively in the following way

$$
\left\{\begin{array}{l}
\left\|\mathbf{F}\left(\mathbf{u}_{l}\right)+\mathbf{J}\left(\mathbf{u}_{l}\right) \delta \mathbf{u}_{l}\right\|<\max \left\{\eta_{a},\left\|\mathbf{F}\left(\mathbf{u}_{l}\right)\right\| \eta_{r}\right\} \\
\mathbf{u}_{l+1}=\mathbf{u}_{l}+\delta \mathbf{u}_{l}, l=0,1, \ldots
\end{array}\right.
$$

for a given initial guess $\mathbf{u}_{0}$. Here, $\mathbf{J}=\frac{\partial \mathbf{F}}{\partial \mathbf{u}}$ is the associated Jacobian matrix, $\eta_{a}$ and $\eta_{r}$ are stopping parameters that determine how accurately the Jacobian system needs to be solved [18].

The Newton iteration is terminated based on the following stopping condition

$$
\left\|\mathbf{F}\left(\mathbf{u}_{l}\right)\right\|<\max \left\{\epsilon_{a},\left\|\mathbf{F}\left(\mathbf{u}_{0}\right)\right\| \epsilon_{r}\right\}
$$

or a sufficiently small Newton update

$$
\left\|\delta\left(\mathbf{u}_{l}\right)\right\|<\epsilon_{s}
$$

where $\epsilon_{a}, \epsilon_{s}$ are absolute tolerances, and $\epsilon_{r}$ is a relative tolerance, respectively.

In (13), we use a right-preconditioned GMRES method to solve the Jacobian system

$$
\mathbf{J M}^{-\mathbf{1}}(\mathbf{M} \delta \mathbf{u})=-\mathbf{F}(\mathbf{u})
$$


with $\mathbf{M}^{-1}$ being a restrictive additive Schwarz preconditioner. To define the preconditioner, we first partition $\Omega$ into $n p$ ( $n p$ is the number of processors) nonoverlapping subdomains $\Omega_{p}, p=1, \ldots, n p$, and pad each subdomain with $\delta$ mesh layers. We denote the overlapping subdomain as $\Omega_{p}^{\delta}$.

For each of the overlapping subdomains we define $\mathbf{J}_{p}$ as the restriction of $\mathbf{J}$ to $\Omega_{p}^{\delta}$, i.e.,

$$
\mathbf{J}_{p}=\mathbf{R}_{p}^{\delta} \mathbf{J}\left(\mathbf{R}_{p}^{\delta}\right)^{\mathrm{T}}
$$

where $\mathbf{R}_{p}^{\delta}$ is the restriction operator.

The restricted additive Schwarz (RAS) method uses residuals from points in the overlapping region, while discarding computed corrections in the overlapping region. It has the standard form:

$$
\mathbf{M}^{-\mathbf{1}}=\sum_{p=1}^{n p}\left(\mathbf{R}_{p}^{0}\right)^{\mathrm{T}} \mathbf{B}_{p}^{-1}\left(\mathbf{R}_{p}^{\delta}\right)
$$

where $\left(\mathbf{R}_{p}^{0}\right)^{\mathrm{T}}$ is the interpolation operator for the nonoverlapping subdomain. The subdomain matrix $\mathbf{B}_{p}$ is crucial to the success of the RAS preconditioner. Here, $\mathbf{B}_{p}^{-1}$ is the inverse or an approximate inverse of $\mathbf{J}_{p}$, whose multiplication with a vector is computed by a sparse LU or ILU factorization. We refer to [12] for a more detailed introduction of RAS.

\section{Numerical results and analysis}

We implement the algorithms described in the previous section on top of the Portable Extensible Toolkit for Scientific computation (PETSc) library from the Argonne National Laboratory [1]. Because of the randomness in the equation, if the problem is solved with two algorithms (for example, an explicit method and an implicit method), they may not arrive at the same steady state solution in the classical sense (i.e., assume the same value at the same spatial location and the same time), but they are considered as the same solution if they agree with each other after certain translations and rotations to be described. To validate the algorithm and software, we first show some 2D experiments in which we compare the implicit results with results obtained with an explicit algorithm. The main focus of this section is the implicit results in $3 \mathrm{D}$.

For the nonlinear solver: the absolute convergence tolerance is $\epsilon_{a}=10^{-8}$; the relative convergence tolerance is $\epsilon_{r}=10^{-6}$; the convergence tolerance in terms of the norm of the change in the solution between steps is $\epsilon_{s}=10^{-7}$. 
For the linear solver, the absolute convergence tolerance is $\eta_{a}=10^{-9}$; the relative convergence tolerance is $\eta_{r}=10^{-3}$; in the RAS preconditioner, sparse LU or ILU decompositions are used as the subdomain solvers; 1 to 3 overlaps are used for different problem sizes and numbers of subdomains.

There are two physical parameters: the interfacial parameter $\epsilon^{2}$, and the amplitude of the thermal fluctuation $\sigma$. The values of $\epsilon^{2}$ and $\sigma$ are varied to demonstrate the system evolution and associated scalability results. For the $\mathrm{CHC}$ equation, we use a constant initial condition $\bar{u}$ (referred to as the volume fraction), since the thermal fluctuation helps drive the steady initial condition into phase separation. Some statistical quantities are necessary to compare numerical solutions. In this paper, we compute the statistical moments from order 2 to 10. Similar to [24], the $q^{\text {th }}$ order moment is defined as:

$$
M_{q}=\int_{\Omega}\left(\frac{u-\bar{u}}{2}\right)^{q} d \Omega,
$$

which is evaluated numerically using the values at the cell centers.

In the next section, $E, E_{1}$, and $E_{2}$ are the free, bulk, and interfacial energy of the system respectively.

\section{1. $2 D$ experiments}

In this section we study the following issues: (1) validation of the fully implicit method and (2) the effect of the thermal fluctuation.

\subsubsection{Validation of the fully implicit method}

To validate the implicit method, we implement a fully explicit method, which has the same discretization in space as the implicit method. Therefore, we evolve the CHC system by the following scheme:

$$
\mathbf{u}^{n}=\mathbf{u}^{n-1}-\Delta t^{n-1}\left(G\left(\mathbf{u}^{n-1}\right)+\zeta_{h}^{n-1}\right) .
$$

For the explicit scheme, we use a constant time step size $\Delta t=10^{-7}$. For the fully implicit scheme, the adaptive time step strategy is applied with an initial time step size $\Delta t=1.5 \times 10^{-4}$.

Fig. 1 shows the final morphologies with and without the thermal fluctuation computed using the fully implicit scheme, and the explicit scheme. Fig. 1(a) is the steady state solution with the thermal fluctuation obtained with the explicit method, and Fig. 1(b) is the corresponding implicit result. They do not look the 
same, in the classical sense; however, if we shift and rotate the final morphologies, they look the same, as pictured in Fig. 1(c). This is due to the periodic boundary conditions and the random initial condition.

For a more comprehensive comparison between the explicit and implicit scheme, Table 1 shows the energies and statistical moments at steady state with and without thermal fluctuation. The values are very close for both schemes.

Table 1: The energies and statistical moments at steady state computed by using the explicit and fully implicit scheme.

\begin{tabular}{|c|c|c|c|c|}
\hline & $E$ & $E_{1}$ & $E_{2}$ & $M_{2}$ \\
\hline explicit & $-1.77 \times 10^{-2}$ & $-3.79 \times 10^{-2}$ & $2.03 \times 10^{-2}$ & $1.24 \times 10^{-1}$ \\
implicit & $-1.77 \times 10^{-2}$ & $-3.79 \times 10^{-2}$ & $2.03 \times 10^{-2}$ & $1.24 \times 10^{-1}$ \\
\hline & $M_{3}$ & $M_{4}$ & $M_{5}$ & $M_{6}$ \\
\hline explicit & $-2.67 \times 10^{-2}$ & $2.45 \times 10^{-2}$ & $-9.80 \times 10^{-3}$ & $6.13 \times 10^{-3}$ \\
implicit & $-2.68 \times 10^{-2}$ & $2.46 \times 10^{-2}$ & $-9.83 \times 10^{-3}$ & $6.15 \times 10^{-3}$ \\
\hline & $M_{7}$ & $M_{8}$ & $M_{9}$ & $M_{10}$ \\
\hline explicit & $-3.01 \times 10^{-3}$ & $1.70 \times 10^{-3}$ & $-8.92 \times 10^{-4}$ & $4.89 \times 10^{-4}$ \\
implicit & $-3.02 \times 10^{-3}$ & $1.70 \times 10^{-3}$ & $-8.95 \times 10^{-4}$ & $4.90 \times 10^{-4}$ \\
& & \multicolumn{3}{|c}{} \\
\hline
\end{tabular}

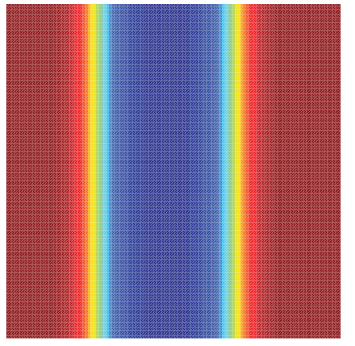

(a)

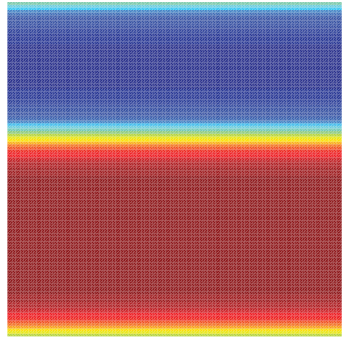

(b)

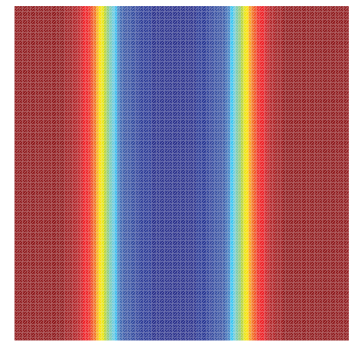

(c)

Figure 1: Steady morphologies: (a) $\sigma=10^{-14}$, explicit scheme ; (b) $\sigma=10^{-14}$, implicit scheme; (c) the translation and rotation of (b).

\subsubsection{Case studies}

Case I. We next study the effect of the thermal fluctuation by showing the evolution of the morphology, energies, and statistical moments with different intensities. We present two test cases with different initial conditions 0.5 and 0.63. For $\bar{u}=0.5$, we set the sharpness parameter $\epsilon^{2}=15 /\left(8 \times 128^{2}\right)$, and for $\bar{u}=0.63$, 
we set $\epsilon^{2}=1 / 12000$, which are the same values used in [24, 37, 39]. We set the intensity parameter to be $\sigma=10^{-14}, 10^{-16}, 10^{-18}, 10^{-20}$. Fig. 2 shows the energies and statistical moments evolution for $\bar{u}=0.5$. We also investigate the morphology evolution in Fig. 3 on a $128 \times 128$ mesh.

It is clear that the increase of the thermal fluctuation intensity leads to an acceleration of system evolution in the early stage. After the initial separation stage, the system starts to coarsen, which is dominated by the distribution of the concentration in the physical domain. The thermal fluctuation not only affects the system positively, but also disturbs the coarsening negatively. Therefore, there is not a monotone relationship between the evolution of the coarsening and the thermal fluctuation. This property can be found in the following examples.
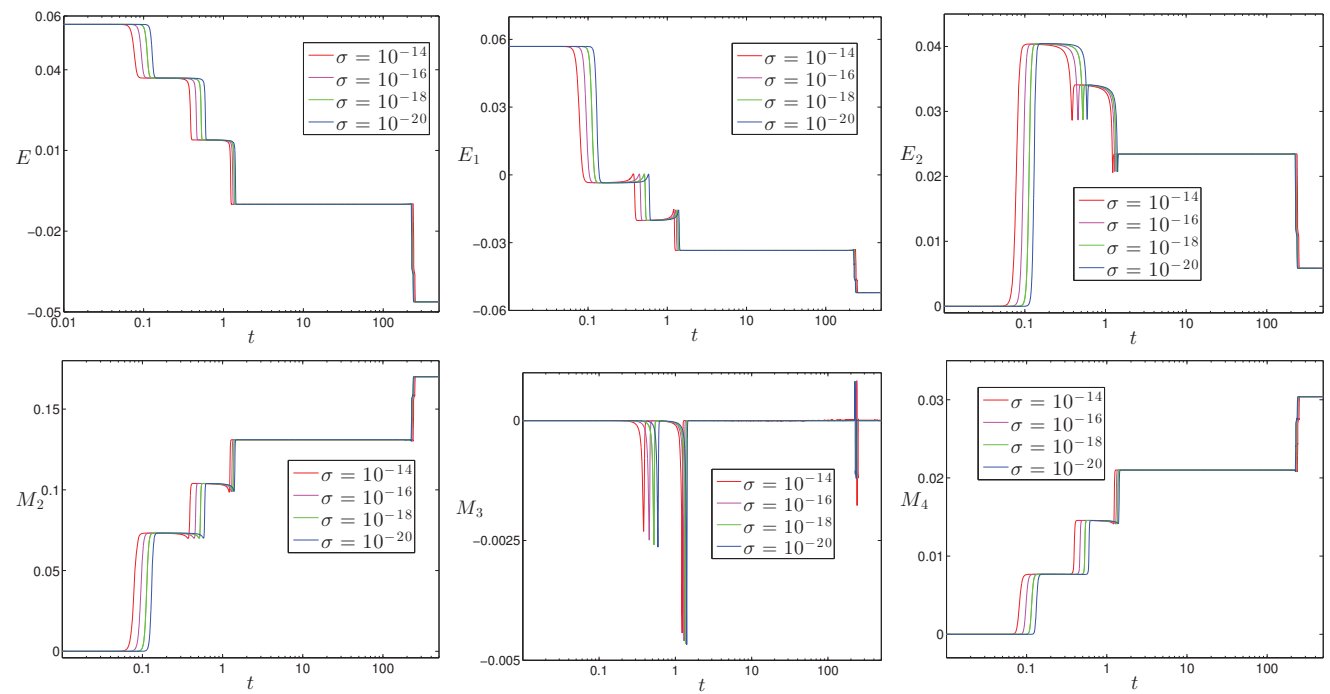

Figure 2: Evolution of the energies and statistical moments for $\epsilon^{2}=15 /\left(8 \times 128^{2}\right), \bar{u}=0$, and $\sigma=10^{-14}, 10^{-16}, 10^{-18}, 10^{-20}$. 


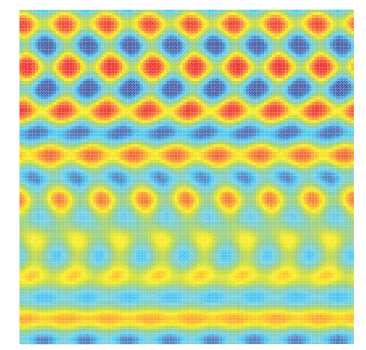

(a) $t=0.05$

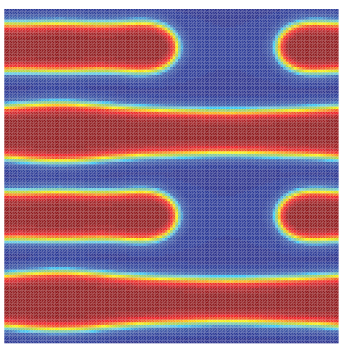

(d) $t=240.0$

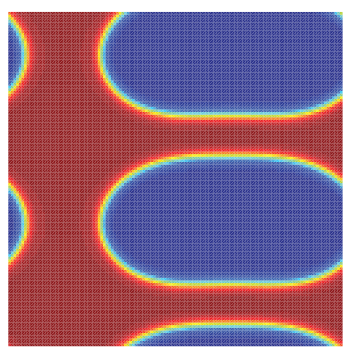

(g) $t=249.8$

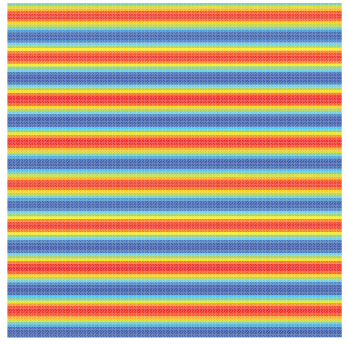

(b) $t=0.1$

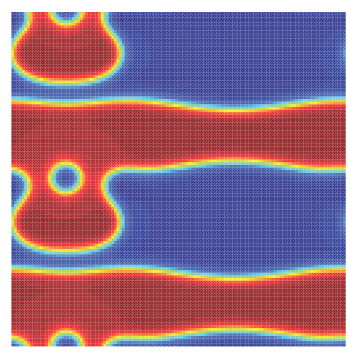

(e) $t=245.0$

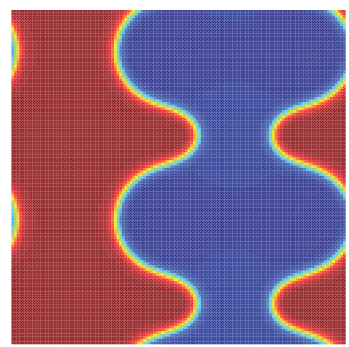

(h) $t=251.4$

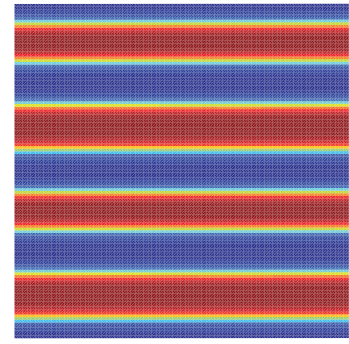

(c) $t=50.0$

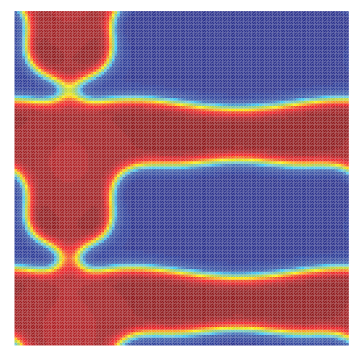

(f) $t=247.2$

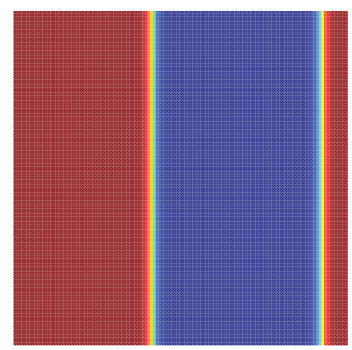

(i) Steady state

Figure 3: Evolution of the concentration difference for $\epsilon^{2}=15 /\left(8 \times 128^{2}\right), \bar{u}=0$, and $\sigma=10^{-14}$.

We next test a case with $\bar{u}=0.63$ on a $256 \times 256$ mesh. Fig. 4 shows the respective evolutions of energies and statistical moments. Fig. 5 is the morphology evolution of the case with $\sigma=10^{-14}$. 

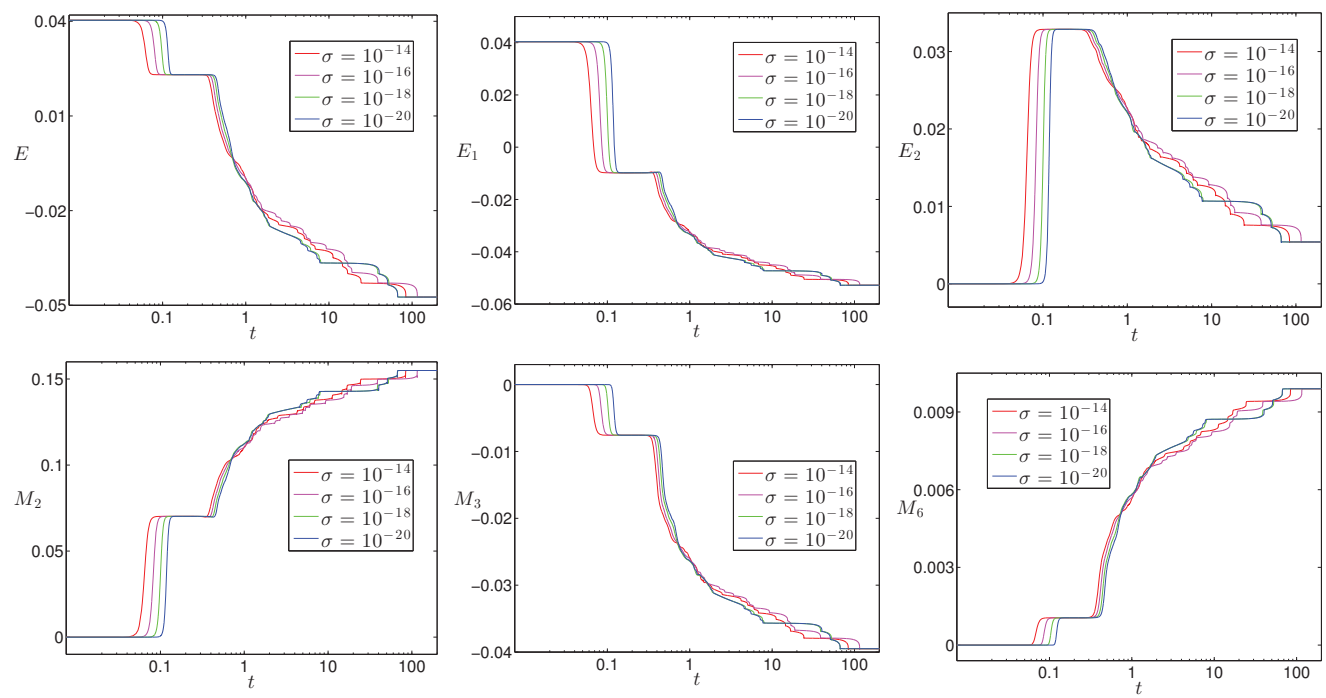

Figure 4: Evolution of the energies and statistical moments for $\epsilon^{2}=1 / 12000, \bar{u}=0.26$, and $\sigma=10^{-14}, 10^{-16}, 10^{-18}, 10^{-20}$. 


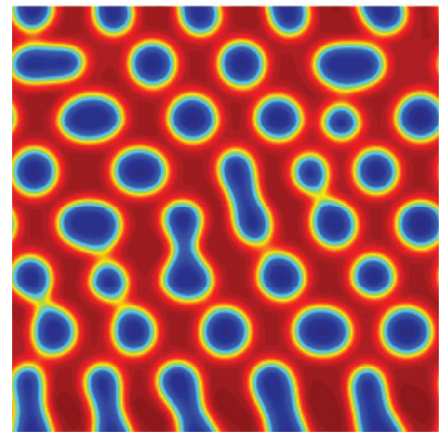

(a) $t=0.5$

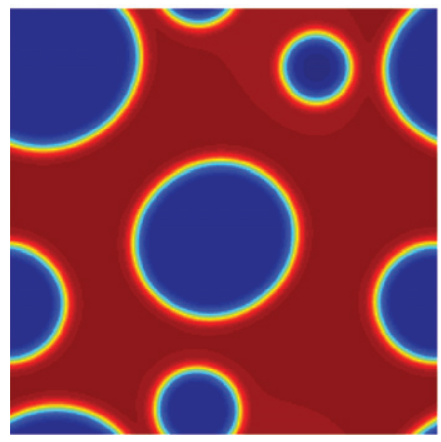

(d) $t=12.5$

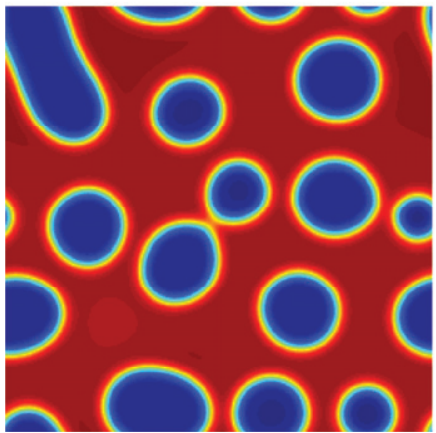

(b) $t=2.0$

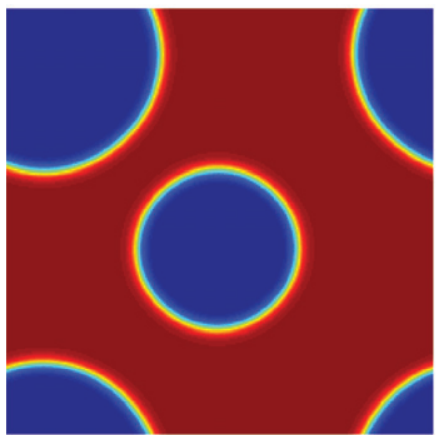

(e) $t=50.0$

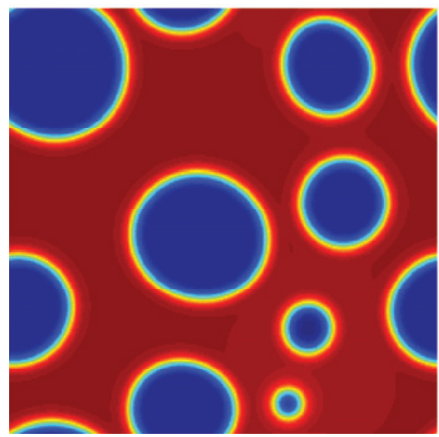

(c) $t=6.0$

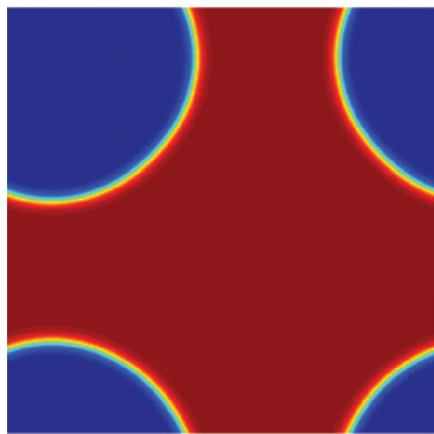

(f) Steady state

Figure 5: Evolution of the concentration difference for $\epsilon^{2}=1 / 12000, \bar{u}=0.26$, and $\sigma=10^{-14}$.

\section{2. $3 D$ experiments}

The study for the $3 \mathrm{D}$ problem is more challenging than the $2 \mathrm{D}$ problem, since the topology of the solution changes significantly as time evolves, and the final steady morphology has more types. For validation, we use the same parameter sets as in [24, 37, 39], which are $\epsilon^{2}=1 / 800, \bar{u}=0.26$, and $\epsilon^{2}=1 / 2400$, $\bar{u}=0.5$. We use the same mesh as in [39], which is $128^{3}$. The system evolution with different intensities of the thermal fluctuation $\sigma=10^{-14}, 10^{-16}$ and $10^{-18}$ is studied. We obtain the same final stages, cylinder and sphere. In terms of energies and statistical moments, our results agree well with the results published in [24, 37]. We also study the equation with other parameter sets and obtain different steady morphologies, e.g., Lawson surface. With some interfacial parameters, we find that the existence of the stochastic noise changes the final morphology, from cylinder to plane. 


\subsubsection{Case studies}

We compute the solution on a $128^{3}$ mesh with four set of parameters, corresponding to four different steady state solutions. We run the tests with different intensities of the thermal fluctuation and compute the energies and statistical moments. We observe that the random noise drives the initially constant concentration to a complex interconnected pattern, and finally a steady morphology.

Case I. In this example, $\bar{u}=0.63, \epsilon^{2}=1 / 800$, and $\sigma=10^{-14}, 10^{-16}, 10^{-18}$. The $\mathrm{CHC}$ equation reaches a steady state and the converged solution is a cylinder. Fig. 6 shows the evolutions of energies and statistical moments, and Fig. 7 is the snapshots of the solution at some intermediate and the final time steps.
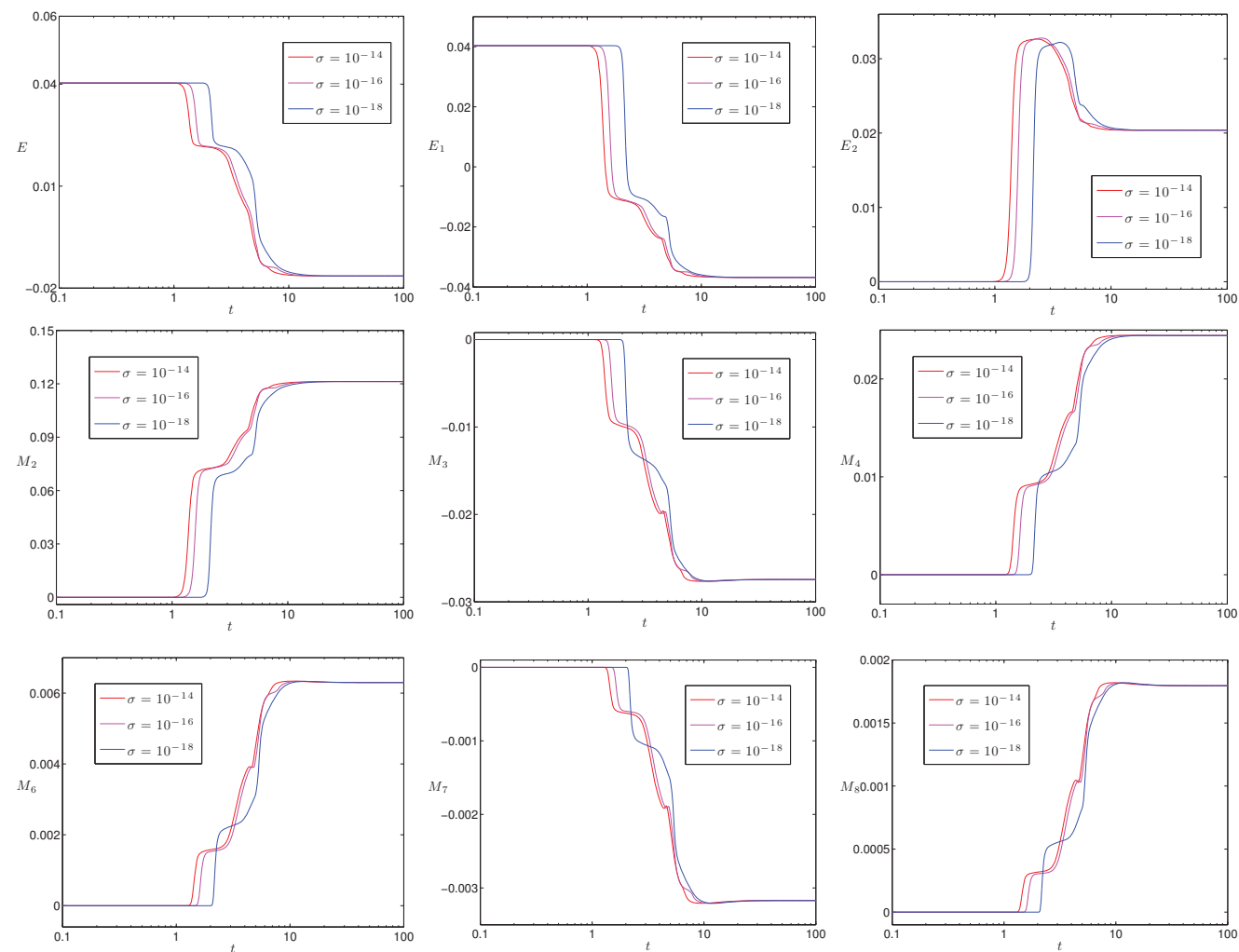

Figure 6: Evolution of the energies and statistical moments for $\epsilon^{2}=1 / 800, \bar{u}=0.26$, and $\sigma=10^{-14}, 10^{-16}, 10^{-18}$. 


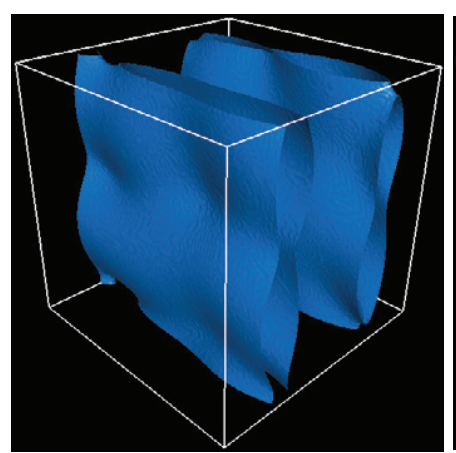

(a) $t=1.7$

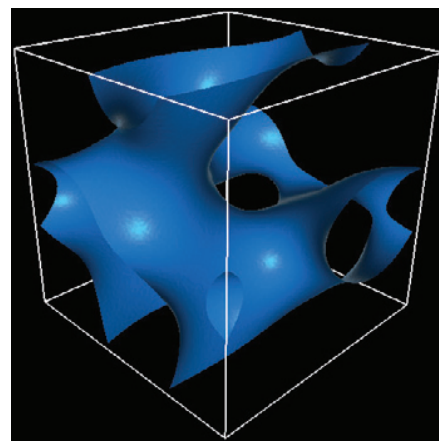

(d) $t=10.0$

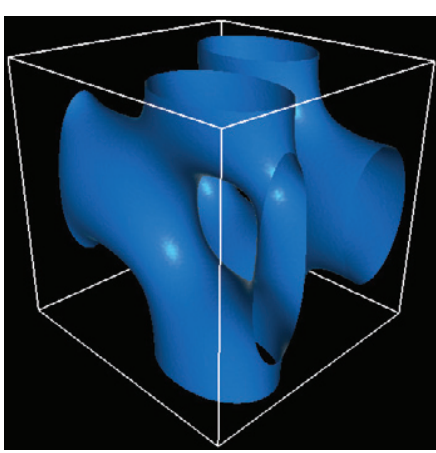

(b) $t=4.0$

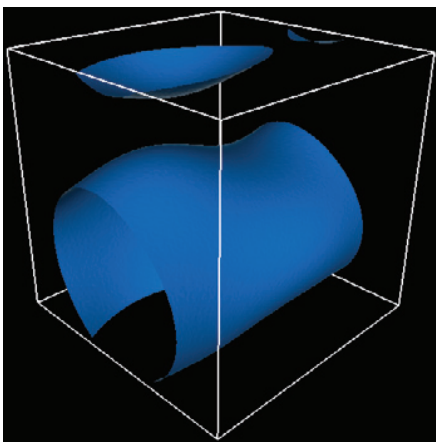

(e) $t=20.0$

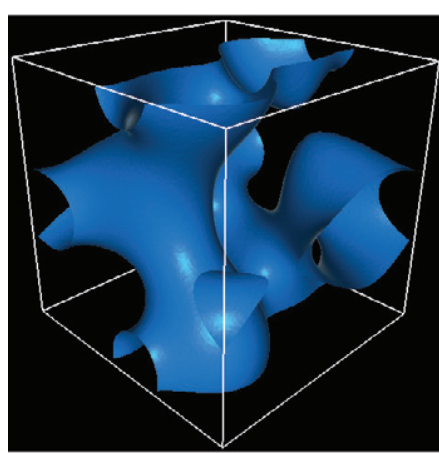

(c) $t=7.0$

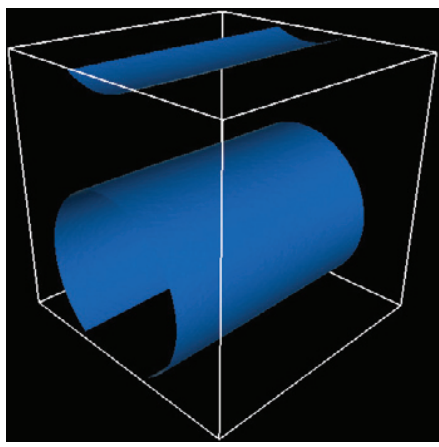

(f) Steady state

Figure 7: Evolution of the concentration difference for $\epsilon^{2}=1 / 800, \bar{u}=0.63$, and $\sigma=10^{-14}$.

Case II. In this example, $\epsilon^{2}=1 / 2400, \bar{u}=0.5$, and $\sigma=10^{-14}, 10^{-16}, 10^{-18}$. See Fig. 8 for energies and statistical moments evolution, and Fig. 9 for the phase variable evolution. The final solution is a sphere. 

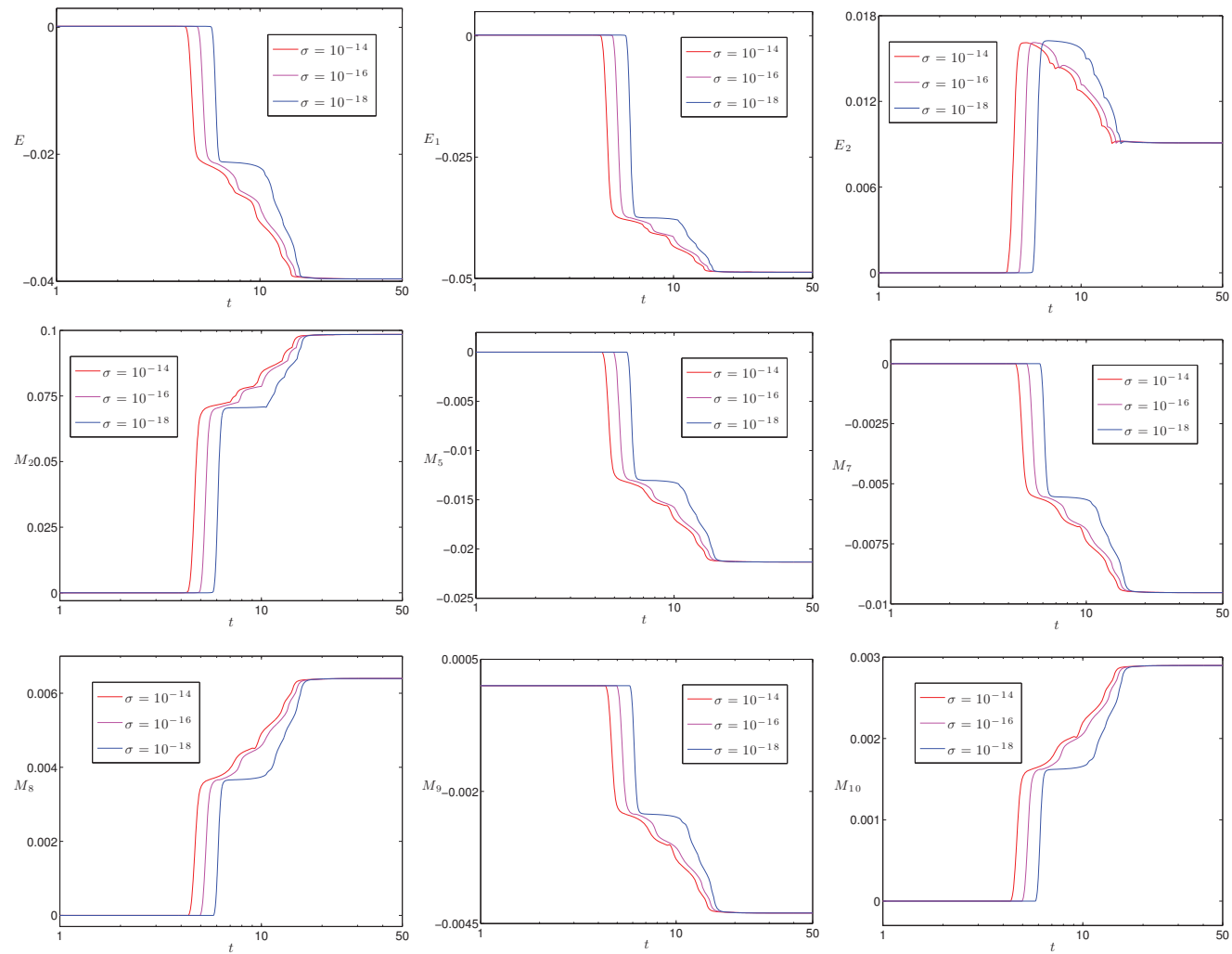

Figure 8: Evolution of the energies and statistical moments for $\epsilon^{2}=1 / 2400, \bar{u}=0.5$, and $\sigma=10^{-14}, 10^{-16}, 10^{-18}$. 


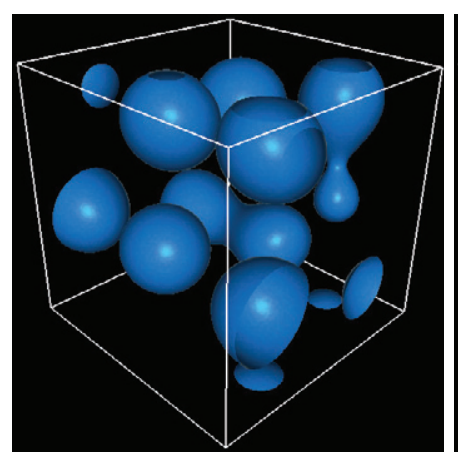

(a) $t=4.0$

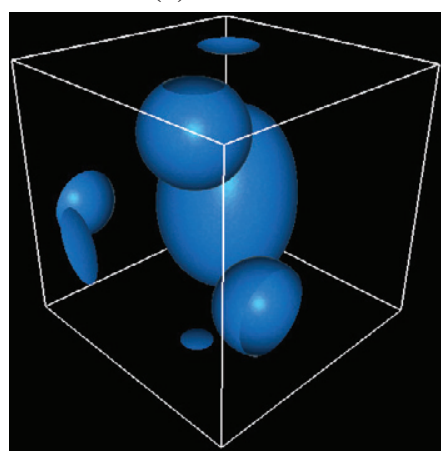

(d) $t=12.0$

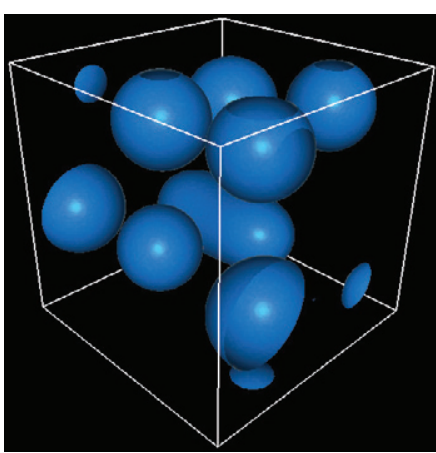

(b) $t=5.0$

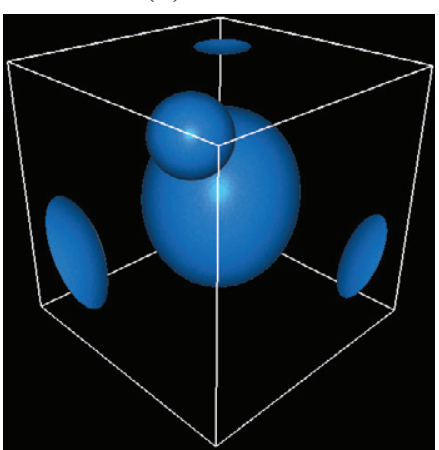

(e) $t=15.0$

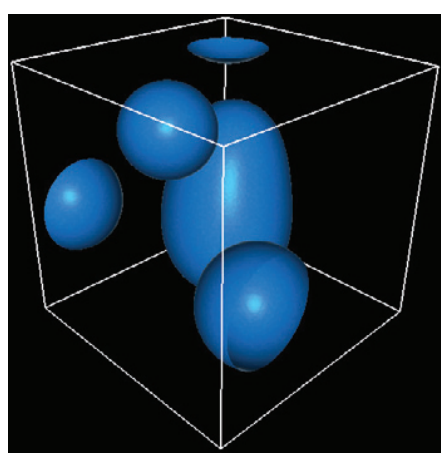

(c) $t=9.0$

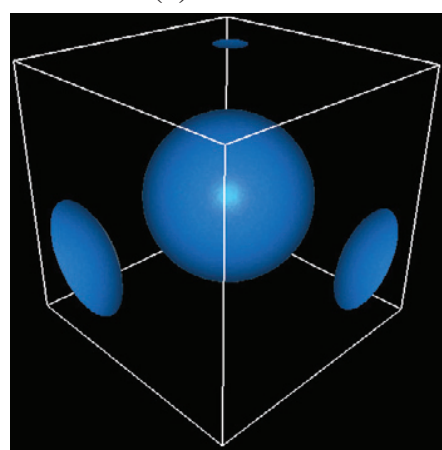

(f) Steady state

Figure 9: Evolution of the concentration difference for $\epsilon^{2}=1 / 2400, \bar{u}=0.5$, and $\sigma=10^{-14}$.

Case III. In this example, we set $\epsilon^{2}=1 / 2400, \bar{u}=0.26$, and $\sigma=10^{-14}, 10^{-16}, 10^{-18}$. Numerical results show that the stochastic forcing does not lead to a different finally morphology than the deterministic PDE. Its solution does evolve to the Lawson surface [37]. Fig. 10 is the evolutions of energies and statistical moments, and Fig. 11 is the snapshots of isosurfaces respectively. Before separating into phases globally, the system finds local metastable configurations as cylinders. As the system evolves, these metastable configurations are destroyed under the influence of the fluctuation. A similar history was observed in two dimensions in Figure 3. 

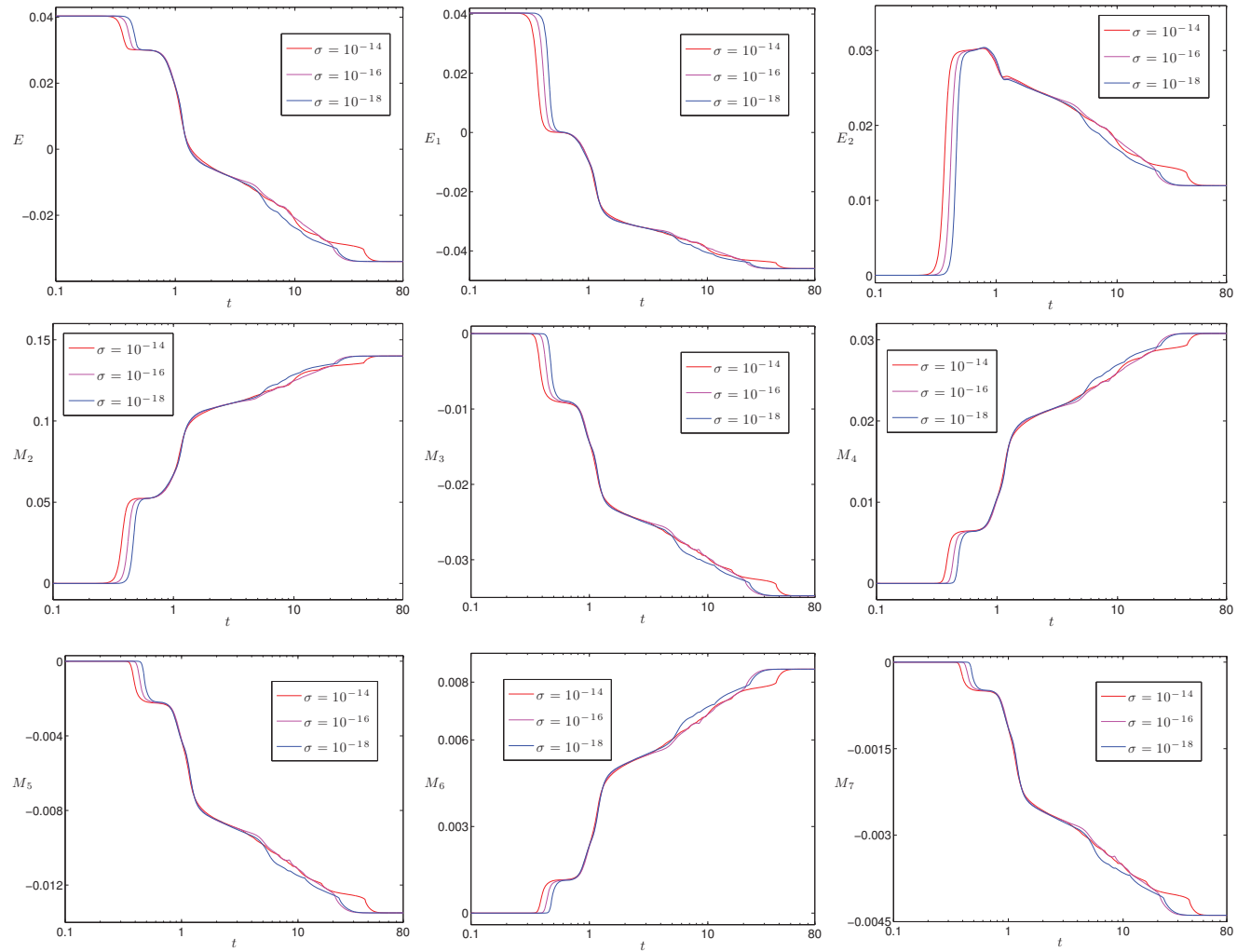

Figure 10: Evolution of the energies and statistical moments for $\epsilon^{2}=1 / 2400, \bar{u}=0.26$, and $\sigma=10^{-14}, 10^{-16}, 10^{-18}$. 


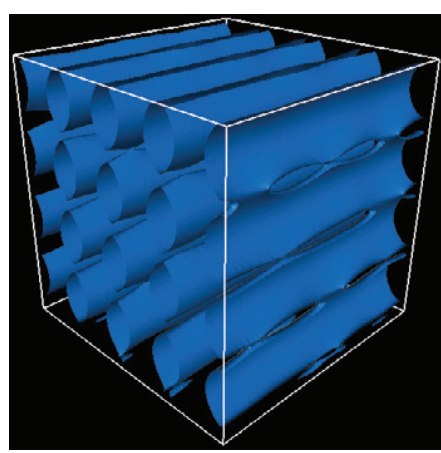

(a) $t=0.5$

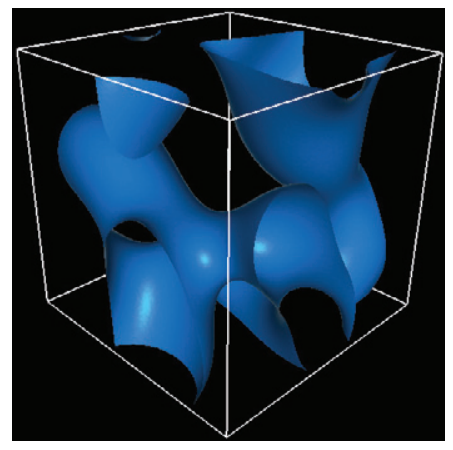

(d) $t=8.0$

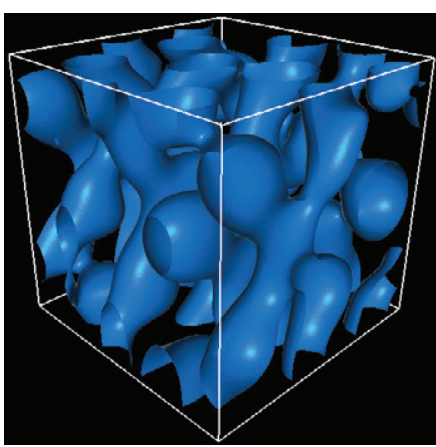

(b) $t=1.0$

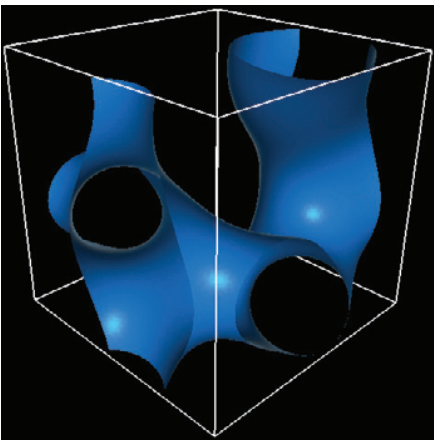

(e) $t=15.0$

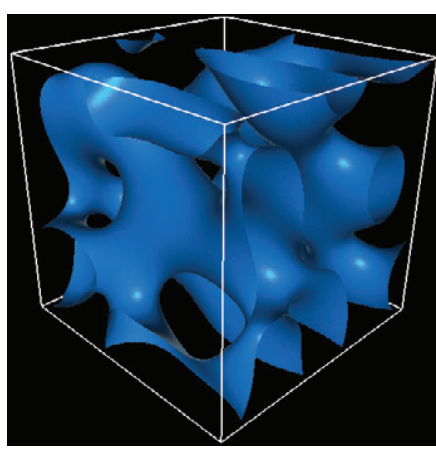

(c) $t=2.0$

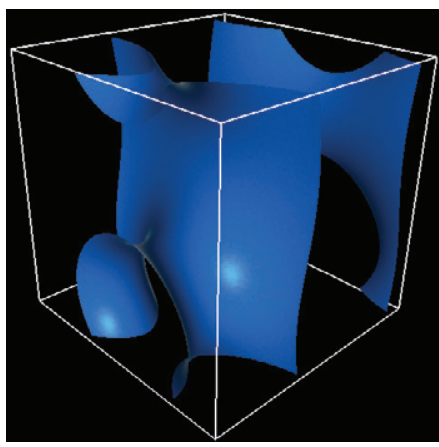

(f) Steady state

Figure 11: Evolution of the concentration difference for $\epsilon^{2}=1 / 2400, \bar{u}=0.26$, and $\sigma=10^{-14}$.

\subsubsection{The adaptive time size evolution}

Our time step control strategy is capable of detecting rare coarsening events that are typical for spinodal decomposition, and adjusting the time step size by several orders of magnitude accordingly. Also, this strategy allows us to reach steady state much faster than using a fixed time step for both two and three dimensional cases. In Fig. 12, we present the history of the time-step size for some examples discussed in the previous subsection. 

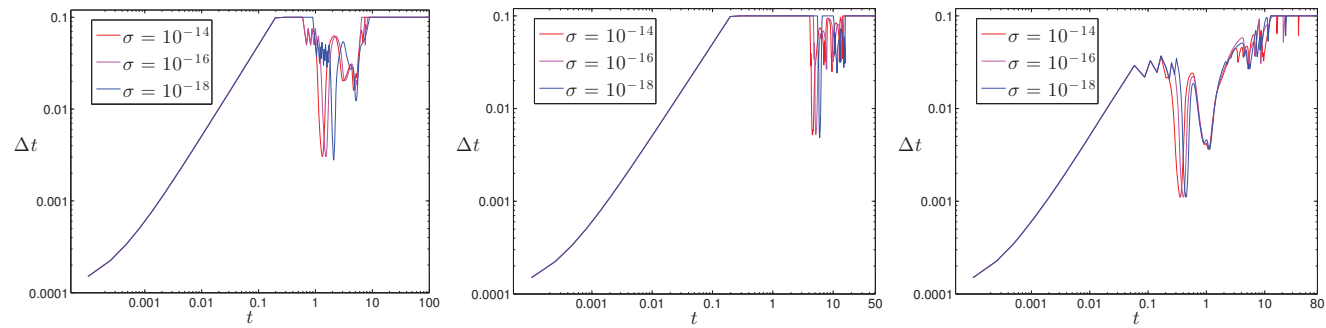

Figure 12: The time size history of Case I, II, and III of 3D experiments.

\subsection{Scalability studies}

In this section, we study the convergence properties of the proposed algorithm. We pay special attention to the number of linear and nonlinear iterations and how they change when the mesh is refined and the number of processors is increased. We also compare the total computing time for each of the test runs. We focus on Case I with $\sigma=10^{-14}$ and 10 fixed-size time steps. We report the performance results at two different points in time; one for the starting time $t=0$, and one for the starting time of the coarsening stage (in this case, the time when the system energy $E=-0.01$ is considered as a typical coarsening time, and test results are in the second part of the following tables).

In tables below, " $n p$ " is the number of processors, "ICT" is the initial coarsening time, "IN" is the number of inexact Newton iterations per time step, "GMRES" is the number of GMRES iterations per Newton step, "Time" is the total execution time in seconds, and "TS" is the time step size. In the tables, the results in the first part is at $t=0$, while the second part is for the coarsening stage.

Table 2 shows a comparison of different subdomain solvers on a $128^{3}$ mesh. It can be seen that when the number of processors is small, ILU is faster than LU, but the scalability is not as good, due to larger number of linear iteration counts. Therefore the optimal choice of the subdomain solver depends on the scale of the problem and the number of processors. 
Table 2: A comparison of different subdomain solvers for Case I. Overlap $=1$, and the time step size $=0.0001$.

\begin{tabular}{|c|c|c|c|c|c|c|c|c|c|c|c|c|}
\hline & \multicolumn{3}{|c|}{$\operatorname{ILU}(0)$} & \multicolumn{3}{|c|}{ ILU(1) } & \multicolumn{3}{|c|}{ ILU(2) } & \multicolumn{3}{|c|}{ LU } \\
\hline \multirow[t]{2}{*}{$n p$} & $\mathrm{NC}$ & GMRE & Time & IN & SMRES & Time & IN C & MRE & S Time & II & MRE & Time \\
\hline & \multicolumn{12}{|c|}{ Average over 10 time steps starting at $t=0$} \\
\hline 256 & 2.0 & 10.0 & .2 & 2.0 & 3.5 & 65.7 & 2.0 & 4.5 & 280.5 & 2.0 & 4.5 & 2401.2 \\
\hline 512 & 0 & 9.6 & & 2.0 & 5.0 & & - & 4.5 & 15 & 13 & 4.5 & 511 \\
\hline 1024 & 2.0 & 9.7 & 27.4 & 2.0 & 9.5 & 30.4 & 2.0 & 4.5 & 105.2 & 2.0 & 4.5 & 169.5 \\
\hline \multirow[t]{2}{*}{2048} & 2.0 & 9.5 & 30.3 & 2.0 & 5.0 & 31.7 & 2.0 & 5.0 & 62.0 & 2.0 & 4.5 & 88.3 \\
\hline & \multicolumn{12}{|c|}{ Average over 10 time steps starting at $t=\mathrm{ICT}$} \\
\hline 256 & 2.0 & 8.0 & 1.3 & 2.0 & 5.0 & 65.8 & 2.0 & 5.0 & 284.6 & 2.2 & 4.3 & 2647.8 \\
\hline 512 & 2.0 & 8.0 & 30.1 & 2.0 & 5.0 & 47.1 & 2.0 & 5.0 & 155.7 & 2.9 & 3.7 & 740.3 \\
\hline 24 & 2.0 & 11.9 & 33.7 & 2.0 & 5.0 & 34.9 & 2.0 & 5.0 & 108.4 & 2.7 & 4.1 & 219.5 \\
\hline 2048 & 2.0 & 12.0 & 34.3 & 2.0 & 5.0 & 31.0 & 2.0 & 4.9 & 63.6 & 2.4 & 4.5 & 105.4 \\
\hline
\end{tabular}

Table 3 shows how the numbers of linear and nonlinear iterations and the computing time change when the mesh is refined and the number of processors increases. The subdomain solver is ILU(0), and the time step size is $10^{-4}$. From the results, we see clearly that the number of nonlinear iterations is not sensitive with respect to the mesh size or the number of processors. Once the mesh size is fixed, the number of linear iterations doesn't change much as we increase the number of processors, but if the number of processors is fixed, then the number of linear iterations increases as we refine the mesh.

Table 3: Iteration numbers of Newton, GMRES, and computing time with different meshes. The subdomain overlap $=1,2,4$, and the subdomain solver is $\operatorname{ILU}(0)$.

\begin{tabular}{|c|ccc|ccc|cc|c|}
\hline & \multicolumn{3}{|c|}{$32^{3}$} & \multicolumn{3}{c|}{$64^{3}$} & \multicolumn{3}{c|}{$128^{3}$} \\
\hline$n p$ & IN GMRES Time & \multicolumn{1}{|c|}{ IN GMRES Time } & IN GMRES Time \\
\hline \multicolumn{6}{|c|}{ Average over 10 time steps starting at $t=0$} \\
\hline 256 & 2.0 & 2.0 & 4.8 & 2.0 & 3.0 & 9.7 & 2.0 & 14.7 & 114.3 \\
\hline 512 & 2.0 & 2.0 & 4.1 & 2.0 & 3.0 & 8.6 & 2.0 & 14.7 & 71.9 \\
\hline 1024 & 2.0 & 2.0 & 4.9 & 2.0 & 3.0 & 14.3 & 2.0 & 19.8 & 74.9 \\
\hline 2048 & 2.0 & 2.0 & 5.9 & 2.0 & 3.0 & 10.3 & 2.0 & 16.7 & 79.8 \\
\hline \multicolumn{6}{|c|}{ Average over 10 time steps starting at $t=\mathrm{ICT}$} \\
\hline 256 & 2.0 & 1.5 & 3.0 & 2.0 & 3.0 & 10.1 & 2.0 & 9.8 & 102.8 \\
\hline 512 & 2.0 & 1.5 & 3.3 & 2.0 & 2.0 & 18.2 & 2.9 & 15.0 & 84.1 \\
\hline 1024 & 2.0 & 1.5 & 4.6 & 2.0 & 2.0 & 8.8 & 2.6 & 9.8 & 60.2 \\
\hline 2048 & 2.0 & 1.5 & 6.8 & 2.0 & 2.5 & 9.2 & 2.3 & 17.0 & 60.5 \\
\hline
\end{tabular}


Table 4 shows the impact of the subdomain overlaps. The subdomain solver is ILU(0), the mesh is $128^{3}$ and the time step size is $10^{-4}$. From the test results, we found that 512 processors are the optimal choice. When we increase the numbers of processors after 512 , the scalability is not linear.

Table 4: Impact of the overlapping size. The subdomain solver is ILU(0), and the time step size $=10^{-4}$.

\begin{tabular}{|c|c|cc|ccc|cc|c|}
\hline & \multicolumn{3}{|c|}{1} & \multicolumn{3}{c|}{2} & \multicolumn{4}{c|}{3} \\
\hline$n p$ & IN GMRES Time & IN GMRES Time & \multicolumn{3}{|c|}{ IN GMRES Time } \\
\hline \multicolumn{6}{|c|}{ Average over 10 time steps starting at $t=0$} \\
\hline 256 & 2.0 & 10.0 & 43.2 & 2.0 & 13.1 & 54.1 & 2.0 & 14.1 & 73.3 \\
\hline 512 & 2.0 & 9.6 & 33.1 & 2.0 & 12.0 & 38.7 & 2.0 & 14.1 & 65.4 \\
\hline 1024 & 2.0 & 9.7 & 27.4 & 2.0 & 11.6 & 53.4 & 2.0 & 14.0 & 45.6 \\
\hline 2048 & 2.0 & 9.5 & 30.3 & 2.0 & 9.6 & 31.9 & 2.0 & 15.5 & 45.3 \\
\hline \multicolumn{6}{|c|}{ Average over 10} & time steps starting at $t=$ ICT \\
\hline 256 & 2.0 & 8.0 & 41.3 & 2.0 & 8.0 & 51.1 & 2.7 & 8.9 & 88.5 \\
\hline 512 & 2.0 & 8.0 & 30.1 & 2.0 & 8.0 & 32.3 & 2.8 & 8.7 & 68.1 \\
\hline 1024 & 2.0 & 11.9 & 33.7 & 3.0 & 6.3 & 70.9 & 2.6 & 8.6 & 55.5 \\
\hline 2048 & 2.0 & 12.0 & 34.3 & 2.0 & 7.5 & 33.9 & 2.6 & 9.0 & 55.9 \\
\hline
\end{tabular}

Table 5 shows a comparison of different time step sizes, on a $128^{3}$ mesh and subdomain solver ILU(0). The results show that the number of linear iterations and computing time increases, as the time step size increases.

Table 5: A comparison of different time step sizes, the subdomain solver is $\operatorname{ILU}(0)$, overlap $=1$.

\begin{tabular}{|c|ccc|ccc|cc|c|c|}
\hline & \multicolumn{3}{|c|}{0.0001} & \multicolumn{3}{c|}{0.001} & \multicolumn{4}{c|}{0.01} \\
\hline$n p$ & IN GMRES Time & IN GMRES Time & IN GMRES Time \\
\hline \multicolumn{8}{|c|}{ Average over 10 time steps starting at $t=0$} \\
\hline 256 & 2.0 & 10.0 & 43.2 & 2.0 & 11.5 & 72.5 & 2.0 & 27.0 & 98.8 \\
\hline 512 & 2.0 & 9.6 & 33.1 & 2.0 & 11.2 & 47.9 & 2.0 & 26.2 & 56.7 \\
\hline 1024 & 2.0 & 9.7 & 27.4 & 2.0 & 11.5 & 37.1 & 2.0 & 26.8 & 43.4 \\
\hline 2048 & 2.0 & 9.5 & 30.3 & 2.0 & 12.0 & 34.5 & 2.0 & 30.3 & 41.1 \\
\hline \multicolumn{8}{|c|}{ Average over 10 time steps starting at $t=$ ICT } \\
\hline 256 & 2.0 & 8.0 & 41.3 & 3.0 & 10.0 & 103.9 & 3.0 & 25.3 & 134.9 \\
\hline 512 & 2.0 & 8.0 & 30.1 & 3.0 & 9.7 & 72.3 & 3.0 & 25.0 & 83.2 \\
\hline 1024 & 2.0 & 11.9 & 33.7 & 3.0 & 10.0 & 52.5 & 3.0 & 26.6 & 64.1 \\
\hline 2048 & 2.0 & 12.0 & 34.3 & 3.0 & 10.0 & 49.9 & 3.0 & 28.8 & 77.1 \\
\hline
\end{tabular}


We remark that when the time step size is large, ILU(0) is not as reliable as ILU(1) and sometime it fails to converge.

\subsection{Comparison with the CH equation}

In this section, we compare the $\mathrm{CHC}$ equation with the $\mathrm{CH}$ equation. For the $\mathrm{CH}$ equation, the constant initial condition keeps the evolution silent forever. Therefore, using the treatment in [24, 37], we apply a random initial condition:

$$
u_{0}(\boldsymbol{x})=\bar{u}+r(\boldsymbol{x})
$$

where $r(\boldsymbol{x})$ is a random variable, with uniform distribution in $[-0.01,0.01]$. In the numerical experiments, an independent random number is generated.

We first solve the $\mathrm{CH}$ equation with parameter sets provided in the previous section for the $\mathrm{CHC}$ equation, including both $2 \mathrm{D}$ and $3 \mathrm{D}$ cases. It is found the $\mathrm{CH}$ and $\mathrm{CHC}$ equation have the same steady state solutions in all tests. Some of these steady states are consistent with other reports [24, 37, 39].

We next solve the $\mathrm{CH}$ and $\mathrm{CHC}$ equation with a set of parameters not used in the previous section, $\epsilon^{2}=1 / 200, \bar{u}=0.26$. It is found that the steady state solution of the $\mathrm{CH}$ equation with the random initial condition is a cylinder, but the steady state solution of the CHC equation is a plane. We also note that the relative percentage difference of the steady system energy between the two models is small (1.5\%). For bulk, interfacial energy, and statistical moments at steady state, the relative percentage difference between the $\mathrm{CH}$ and $\mathrm{CHC}$ equation are larger (from $4.5 \%$ to $77 \%$ ). Fig. 13 illustrates the steady states of the $\mathrm{CHC}$ and $\mathrm{CH}$ models respectively. 


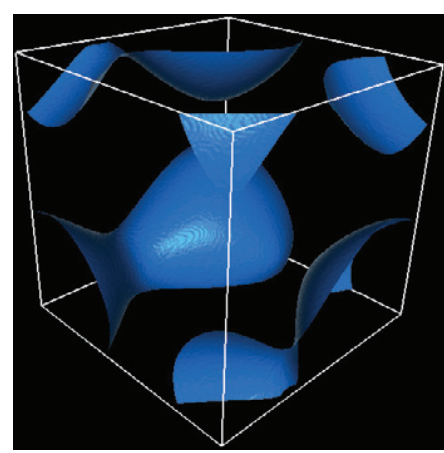

(a) $t=4.0$

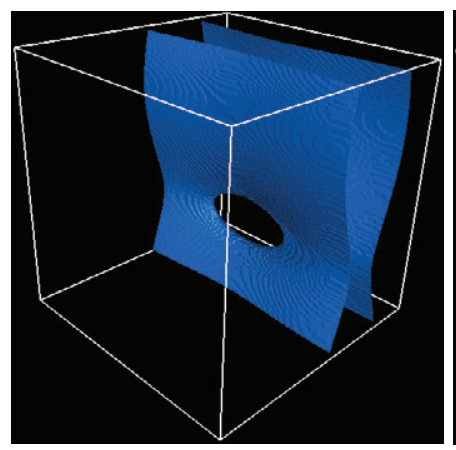

(d) $t=6.8$

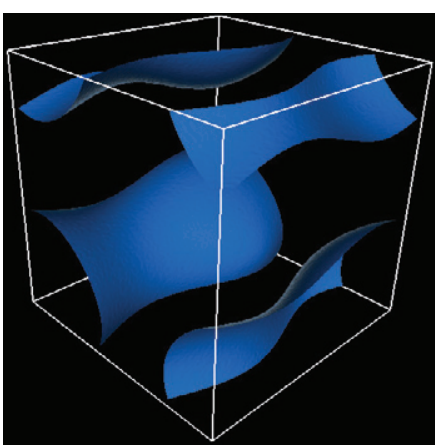

(b) $t=6.0$

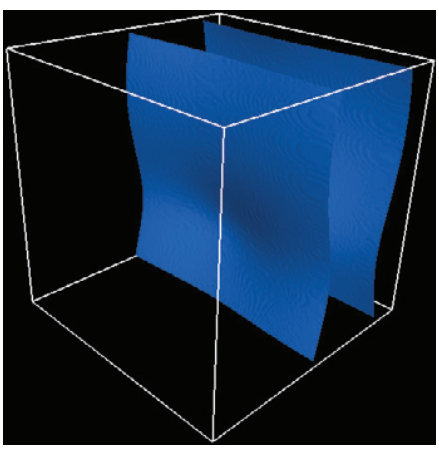

(e) $t=7.7$

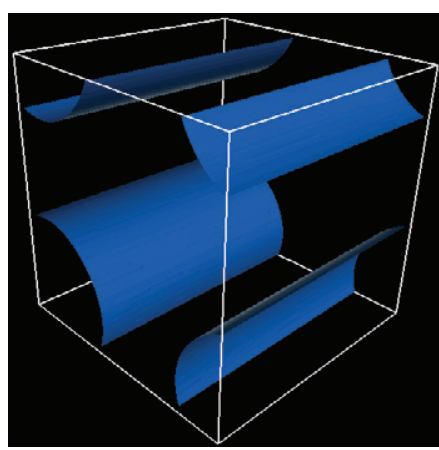

(c) Steady state

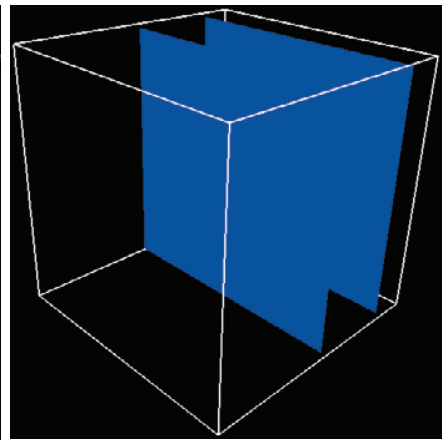

(f) Steady state

Figure 13: Evolution of the concentration difference computed with the $\mathrm{CH}$ equation with the random initial condition (the top row) and the $\mathrm{CHC}$ equation with the random noise $\sigma=10^{-14}$ (the bottom row), $\epsilon^{2}=1 / 200, \bar{u}=0.26$.

\section{Conclusions}

In this paper, we presented a fully implicit method for solving the CahnHilliard phase-field problem with the thermal fluctuation. Our method consists of a fully implicit cell-centered finite difference discretization, together with an adaptive time stepping strategy. For solving the nonlinear system of equations at each time step, we use an NKS algorithm, which proves to be effective for this highly nonlinear problem. We are able to obtain steady state solutions in both $2 \mathrm{D}$ and $3 \mathrm{D}$.

Typical steady state morphologies, e.g., circle, slab, cylinder, are obtained. We found that the random noise, the interfacial parameter and the initial concentration have significant impact on the steady state solution. For certain parameter sets, the $\mathrm{CHC}$ equation and the $\mathrm{CH}$ equation evolve to different steady states. The 
evolution of energies and statistical moments with different intensities of thermal fluctuation are also studied. We found that the thermal fluctuation plays an important role in the early stage, which is consistent with Cook's conclusion. The increase of the thermal fluctuation intensity leads to the acceleration of the phase separation. Later, during the coarsening stage, the separated concentration distribution dominates the slow process. There is no monotonic relationship between the system evolution and the thermal fluctuation in the coarsening process. To our knowledge, this is the first time that 3D steady state calculations of the CHC equation are carried out. We also studied the scalability of our method with respect to the number of processors, the mesh size, subdomain solver, subdomain overlap, and time step size.

Our future research includes the extension of the Schwarz methods to multi levels so that higher resolution meshes can be used on machines with larger number of processors.

\section{Acknowledgement}

The authors wish to thank Professor Marc Spiegelman and Professor Yuefan Deng for many helpful discussions. This work was supported by the U.S. Department of Energy (under Contract No. DE-FC02-06ER25784). Their support is gratefully acknowledged.

[1] S. Balay, J. Brown, K. Buschelman, V. Eijkhout, W. Gropp, D. Kaushik, M. Knepley, L.C. McInnes, B. Smith, and H. Zhang, PETSc Users Manual, Tech. Rep. ANL-95/11 - Revision 3.4, Argonne National Laboratory (2013).

[2] J.W. Barrett, J.F. Blowey, Modelling and visualizing the Cahn-Hilliard-Cook equation, Math. Comp. 68 (1999) 487-517.

[3] K. Binder, Kinetics of phase separation. In: Stochastic nonlinear systems in physics, chemistry, and biology, Springer Series in Synergetics, BerlinHeidelberg-New York: Springer 8 (1981) 62-71.

[4] D. Blömker, S. Maier-paape, T. Wanner, Spinodal decomposition for the Cahn-Hilliard-Cook equation, Comm. Math. Phys., 223 (2001) 553-582.

[5] D. Blömker, S. Maier-paape, T. Wanner, Second Phase spinodal decomposition for the Cahn-Hilliard-Cook equation, Trans. American Math. Soc., 360 (2007) 449-489. 
[6] G.E.P. Box, M.E. Muller, A note on the generation of random normal deviates, Ann. Math. Stat. 29 (1958) 610-611.

[7] J.W. Cahn, On spinodal decomposition, Acta Metall. 9 (1961) 795-801.

[8] J.W. Cahn, J.E. Hilliard, Free energy of a nonuniform system. I: Interfacial energy, J. Chem. Phys. 28 (1958) 258-267.

[9] J.W. Cahn, J.E. Hilliard, Free energy of a nonuniform system. II: Thermodynamic basis, J. Chem. Phys. 30 (1959) 1121-1135.

[10] X.-C. Cai, W.D. Gropp, D.E. Keyes, R.G. Melvin, Parallel Newton-KrylovSchwarz algorithms for the transonic full potential equation, SIAM J. Sci. Comput. 19 (1998) 246-265.

[11] X.-C. Cai, W.D. Gropp, D.E. Keyes, M.D. Tidriri, Newton-Krylov-Schwarz methods in CFD, Proceedings of the International Workshop on the NavierStokes Equations, Notes in Numerical Fluid Mechanics, R. Rannacher, eds. Vieweg Verlag, Braunschweig, (1994).

[12] X.-C. Cai, M. Sarkis, A restricted additive Schwarz preconditioner for general sparse linear systems, SIAM J. Sci. Comput. 21 (1999) 792-797.

[13] C. Cardon-Weber, Implicit approximation scheme for the Cahn-Hilliard stochastic equation, Prépublication 613 du Laboratoire de Probabilités et Modèles Aléatoires (2000).

[14] S.M. Choo, S.K. Chung, K.I. Kim, Conservative nonlinear difference scheme for the Cahn-Hilliard equation II, Comput. Math. Appl. 39 (2000) 229-243.

[15] H.E. Cook, Brownian motion in spinodal decomposition, Acta Metall. 18 (1970) 297-306.

[16] L. Cueto-Felgueroso, J. Peraire, A time-adaptive finite volume method for the Cahn-Hilliard and Kuramoto-Sivashinsky equations, J. Comput. Phys. 227 (2008) 9985-10017.

[17] I.C. Dolcetta, S.F. Vita, R. March, Area preserving curve shortening flows: from phase transitions to image processing, Interfaces Free Boundaries 4 (2002) 325-343. 
[18] S.C. Eisenstat, H.F. Walker, Choosing the forcing terms in an inexact Newton method, SIAM J. Sci. Comput. 17 (1996) 16-32.

[19] K.R. Elder, T.M. Rogers, R.C. Desai, Numerical study of the late stages of spinodal decomposition, Phys. Rev. B 37 (1987) 9638-9649.

[20] K.R. Elder, T.M. Rogers, R.C. Desai, Early stages of spinodal decomposition for the Cahn-Hilliard-Cook model of phase separation, Phys. Rev. B 38 (1988) 4725-4739.

[21] C.M. Elliott, D.A. French, A nonconforming finite-element method for the two-dimensional Cahn-Hilliard equation, SIAM J. Numer. Anal. 26 (1989) 884-903.

[22] C.M. Elliot, D.A. French, F. Milner, A second order splitting method for the Cahn-Hilliard equation, Numer. Math. 54 (1989) 575-590.

[23] P.K. Galenko, D. Kharchenko, I. Lysenko, Stochastic generalization for a hyperbolic model of spinodal decomposition, Physica A 389 (2010) 34433455 .

[24] H. Gomez, V.M. Calo, Y. Bazilevs, T.J.R. Hughes, Isogeometric analysis of the Cahn-Hilliard phase-field model, Comp. Meth. App. Mech. Eng. 197 (2008) 4333-4352.

[25] K.A. Hawick, D.P. Playne, Modeling and visualizing the Cahn-HilliardCook equation, Proceedings of 2008 International Conference on Modeling, Simulation and Visualization Methods (2008).

[26] D.K. Kaushik, D.E. Keyes, B.F. Smith, Newton-Krylov-Schwarz methods for aerodynamics problems: compressible and incompressible flows on unstructured grids, Proceedings of the 11th International Conference on Domain Decomposition Methods (1999) 513-520.

[27] J. Kim, A numerical method for the Cahn-Hilliard equation with a variable mobility, Comm. Nonlinear Sci. Numer. Simul. 12 (2007) 1560-1571.

[28] J. Kim, K. Kang, A numerical method for the ternary Cahn-Hilliard system with a degenerate mobility, Appl. Numer. Math. 59 (2009) 1029-1042.

[29] M. Kovács, S. Larsson, A. Mesforush, Finite element approximation of the Cahn-Hilliard-Cook equation, SIAM J. Numer. Anal., 49 (2011) 2407-2429. 
[30] J.S. Langer, M. Bar-on, H.D. Miller, New computational method in the theory of spinodal decomposition, Phys. Rev. A 11 (1975) 1417-1429.

[31] A. Milchev, D.W. Heermann, K. Binder, Monte-Carlo simulation of the Cahn-Hilliard model of spinodal decomposition, Acta. Metall. 36 (1988) 377-383.

[32] W. Mulder, B. Van Leer, Experiments with implicit upwind methods for the Euler equations, J. Comput. Phys. 59 (1985).

[33] R. Saxena, G.T. Caneba, Studies of spinodal decomposition in a ternary polymer-solvent-nonsolvent systems, Polym. Eng. Sci. 42 (2002) 10191031.

[34] D. Saylor, C.S. Kim, D. Patwardhan, J. Warren, Diffuse-interface theory for structure formation and release behavior in controlled drug release systems, Acta Biomater. 3 (2007) 851-864.

[35] C. Shen, J. Simmons, Y. Wang, Effect of elastic interaction on nucleation: II. Implementation of strain energy of nucleus formation in the phase field method, Acta. Materialia 55 (2007) 1457-1466.

[36] S. Tremaine, On the origin of irregular structure in Saturn's rings, Astro. J. 125 (2003) 894-901.

[37] O. Wodo, B. Ganapathysubramanian, Computationally efficient solution to the Cahn-Hilliard equation: adaptive implicit time schemes, mesh sensitivity analysis and the 3D isoperimetric problem, J. Comput. Phys. 230 (2011) 6037-6060.

[38] O. Wodo, B. Ganapathysubramanian, Modeling morphology evolution during solvent-based fabrication of organic solar cells, Comput. Mater. Sci. 55 (2012) 113-126.

[39] C. Yang, X.-C. Cai, D.E. Keyes, M. Pernice, Parallel domain decomposition methods for the 3D Cahn-Hilliard equation, SciDAC 2011 (2011).

[40] C. Yang, X.-C. Cai, D.E. Keyes, M. Pernice, Newton-Krylov-Schwarz method for a coupled Allen-Cahn/Cahn-Hilliard system, Proceedings of the 21th International Conference on Domain Decomposition Methods (2012). 
[41] H. Yu, W. Lu, Ordering of nanovoids in an anisotropic solid driven by surface misfit, J. Comput. Theor. Nano. 2 (2005) 256-262.

[42] S. Zhang, M. Wang, A nonconforming finite element method for the CahnHilliard equation, J. Comput. Phys. 229 (2010) 7361-7372.

[43] B. Zhou, A. Powell, Phase field simulation of early stage structure formation during immersion precipitation of polymeric membranes in $2 \mathrm{D}$ and $3 \mathrm{D}, \mathrm{J}$. Membr. Sci. 268 (2006) 150-164. 